\title{
SYMMETRIC POLYNOMIALS IN THE 3D FOURIER EQUATION
}

\author{
Grzegorz Biernat, Sylwia Lara-Dziembek, Edyta Pawlak \\ Institute of Mathematics, Czestochowa University of Technology \\ Czestochowa, Poland \\ grzegorz.biernat@im.pcz.pl,sylwia.lara@im.pcz.pl,edyta.pawlak@im.pcz.pl
}

\begin{abstract}
The work is a continuation of the method of calculating the determinant of the block matrix in the three-dimensional case. In this article the symmetric polynomials are used.
\end{abstract}

Keywords: block matrices, symmetric polynomials

\section{Introduction}

In this paper we return to the development of the determinant of the block matrix in the $3 \mathrm{D}$ case. We express this determinant by the symmetric polynomials.

The considered problem concerns the effective formulas expressing the symmetric polynomials of three groups of variables by the symmetric polynomials due to each of these groups.

\section{Solution of the problem}

The heat flow in the 3D domain is described by the Fourier equation

$$
\lambda\left(\frac{\partial^{2} T(x, y, z, t)}{\partial x^{2}}+\frac{\partial^{2} T(x, y, z, t)}{\partial y^{2}}+\frac{\partial^{2} T(x, y, z, t)}{\partial z^{2}}\right)=\rho c \frac{\partial T(x, y, z, t)}{\partial t}
$$

where $\lambda$ is a thermal conductivity, $c$ is a specific heat, $\rho$ is a mass density and $T, x$, $y, z, t$ denote the temperature, geometrical co-ordinates and time.

Assuming the following difference quotients we get the differential approximation of the second derivatives appearing in the equation (1) 


$$
\begin{array}{ll}
\frac{\Delta^{2} T}{\Delta x^{2}}=\frac{T_{i-1, j, k, l}-2 T_{i, j, k, l}+T_{i+1, j, k, l}}{(\Delta x)^{2}}, & 1 \leq i \leq m-1 \\
\frac{\Delta^{2} T}{\Delta y^{2}}=\frac{T_{i, j-1, k, l}-2 T_{i, j, k, l}+T_{i, j+1, k, l}}{(\Delta y)^{2}}, & 1 \leq j \leq n-1 \\
\frac{\Delta^{2} T}{\Delta z^{2}}=\frac{T_{i, j, k-1, l}-2 T_{i, j, k, l}+T_{i, j, k+1, l}}{(\Delta z)^{2}}, & 1 \leq k \leq p-1
\end{array}
$$

and the approximation of the first derivative of the time

$$
\frac{\Delta T}{\Delta t}=\frac{T_{i, j, k, l}-T_{i, j, k, l-1}}{\Delta t}, \quad 1 \leq l \leq q
$$

The internal iterations taking the following differential form

$$
\lambda\left(\frac{\Delta^{2} T}{\Delta x^{2}}+\frac{\Delta^{2} T}{\Delta y^{2}}+\frac{\Delta^{2} T}{\Delta z^{2}}\right)=\rho c \frac{\Delta T}{\Delta t}
$$

The Finite Difference Method leads to the internal system of equations

$$
\begin{aligned}
& \frac{\lambda}{(\Delta x)^{2}} T_{i-1, j, k, l}-\frac{2 \lambda}{(\Delta x)^{2}} T_{i, j, k, l}+\frac{\lambda}{(\Delta x)^{2}} T_{i+1, j, k, l}+ \\
+ & \frac{\lambda}{(\Delta y)^{2}} T_{i, j-1, k, l}-\frac{2 \lambda}{(\Delta y)^{2}} T_{i, j, k, l}+\frac{\lambda}{(\Delta y)^{2}} T_{i, j+1, k, l}+ \\
+ & \frac{\lambda}{(\Delta z)^{2}} T_{i, j, k-1, l}-\frac{2 \lambda}{(\Delta z)^{2}} T_{i, j, k, l}+\frac{\lambda}{(\Delta z)^{2}} T_{i, j, k+1, l}= \\
= & \frac{\rho c}{\Delta t} T_{i, j, k, l}-\frac{\rho c}{\Delta t} T_{i, j, k, l-1}
\end{aligned}
$$

in each time step $l$.

The determinant of the matrix $A_{3}$ [1]

$$
A_{3}=\left[\begin{array}{rrrrrrr}
A_{2} & I_{2} & \ldots & \ldots & \ldots & \ldots & \ldots \\
I_{2} & A_{2} & I_{2} & \ldots & \ldots & \ldots & \ldots \\
\vdots & \vdots & \vdots & \ddots & \vdots & \vdots & \vdots \\
\ldots & \ldots & \ldots & \ldots & I_{2} & A_{2} & I_{2} \\
\ldots & \ldots & \ldots & \ldots & \ldots & I_{2} & A_{2}
\end{array}\right]_{p \times p \text { (block dimension) }}
$$


where

$$
A_{2}=\left[\begin{array}{rrrrrrr}
A_{1} & I_{1} & \ldots & \ldots & \ldots & \ldots & \ldots \\
I_{1} & A_{1} & I_{1} & \ldots & \ldots & \ldots & \ldots \\
\vdots & \vdots & \vdots & \ddots & \vdots & \vdots & \vdots \\
\ldots & \ldots & \ldots & \ldots & I_{1} & A_{1} & I_{1} \\
\ldots & \ldots & \ldots & \ldots & \ldots & I_{1} & A_{1}
\end{array}\right]_{n \times n \text { (block dimension) }}
$$

and

$$
I_{2}=\left[\begin{array}{rrrrrrr}
I_{1} & 0 & \ldots & \ldots & \ldots & \ldots & \ldots \\
0 & I_{1} & 0 & \ldots & \ldots & \ldots & \ldots \\
\vdots & \vdots & \vdots & \ddots & \vdots & \vdots & \vdots \\
\ldots & \ldots & \ldots & \ldots & 0 & I_{1} & 0 \\
\ldots & \ldots & \ldots & \ldots & \ldots & 0 & I_{1}
\end{array}\right]_{n \times n \text { (block dimension })}
$$

is given by the formula

$$
\operatorname{det} A_{2}=\operatorname{det}\left[A_{1}^{n}-\left(\begin{array}{c}
n-1 \\
1
\end{array}\right) A_{1}^{n-2}+\left(\begin{array}{c}
n-2 \\
2
\end{array}\right) A_{1}^{n-4}-\ldots\right]
$$

In the formula (7) we have

$$
A_{1}=\left[\begin{array}{rrrrrrr}
a & 1 & \ldots & \ldots & \ldots & \ldots & \ldots \\
1 & a & 1 & \ldots & \ldots & \ldots & \ldots \\
\vdots & \vdots & \vdots & \ddots & \vdots & \vdots & \vdots \\
\ldots & \ldots & \ldots & \ldots & 1 & a & 1 \\
\ldots & \ldots & \ldots & \ldots & \ldots & 1 & a
\end{array}\right]_{m \times m}
$$

and

$$
I_{1}=\left[\begin{array}{rrrrrrr}
1 & 0 & \ldots & \ldots & \ldots & \ldots & \cdots \\
0 & 1 & 0 & \ldots & \ldots & \ldots & \ldots \\
\vdots & \vdots & \vdots & \ddots & \vdots & \vdots & \vdots \\
\cdots & \ldots & \ldots & \ldots & 0 & 1 & 0 \\
\ldots & \ldots & \ldots & \ldots & \ldots & 0 & 1
\end{array}\right]_{m \times m}
$$

We consider only the standardized case $\left(b_{1}=1\right.$ in [2]) with the condition $\operatorname{det} A_{1} \neq 0$. We apply the polynomials 
- $f$ of degree $n$

$$
\begin{aligned}
& f(x)=x^{n}-\left(\begin{array}{c}
n-1 \\
1
\end{array}\right) x^{n-2}+\left(\begin{array}{c}
n-2 \\
2
\end{array}\right) x^{n-4}-\ldots= \\
& =\left(x-p_{1}\right)\left(x-p_{2}\right)\left(x-p_{3}\right) \ldots \cdot\left(x-p_{n}\right)
\end{aligned}
$$

- $\quad g$ of degree $p$

$$
\begin{aligned}
& g(y)=y^{p}-\left(\begin{array}{c}
p-1 \\
1
\end{array}\right) y^{p-2}+\left(\begin{array}{c}
p-2 \\
2
\end{array}\right) y^{p-4}-\ldots= \\
& =\left(y-q_{1}\right)\left(y-q_{2}\right)\left(y-q_{3}\right) \cdot \ldots \cdot\left(y-q_{p}\right)
\end{aligned}
$$

and then we obtain

$$
\begin{aligned}
& \operatorname{det} A_{3}=\operatorname{det}\left[\left(A_{2}-q_{1} I_{2}\right)\left(A_{2}-q_{2} I_{2}\right)\left(A_{2}-q_{3} I_{2}\right)\right. \\
& =\underbrace{\operatorname{det}\left(A_{2}-q_{1} I_{2}\right)}_{W_{A_{2}}\left(q_{1}\right)} \cdot \underbrace{\operatorname{det}\left(A_{2}-q_{2} I_{2}\right)}_{W_{A_{2}}\left(q_{2}\right)} \cdot \underbrace{\operatorname{det}\left(A_{2}-q_{3} I_{2}\right)}_{W_{A_{2}}\left(q_{3}\right)} \cdot \ldots \cdot \underbrace{\operatorname{det}\left(A_{2}-q_{p} I_{2}\right)}_{\left.\left.W_{A_{1}} I_{2}\right)\right]=}= \\
& =\underbrace{\operatorname{det}\left[A_{1}-\left(p_{1}+q_{1}\right) I_{1}\right]}_{W_{A_{1}}\left(p_{1}+q_{1}\right)} \cdot \underbrace{\operatorname{det}\left[A_{1}-\left(p_{1}+q_{2}\right) I_{1}\right]}_{W_{A_{1}}\left(p_{1}+q_{2}\right)} \cdot \ldots \cdot \underbrace{\operatorname{det}\left[A_{1}-\left(p_{1}+q_{p}\right) I_{1}\right]}_{W_{A_{1}}\left(p_{1}+q_{p}\right)} . \\
& =\underbrace{\operatorname{det}\left[A_{1}-\left(p_{n}+q_{1}\right) I_{1}\right]}_{W_{A_{1}}\left(p_{n}+q_{1}\right)} \cdot \underbrace{\operatorname{det}\left[A_{1}-\left(p_{n}+q_{2}\right) I_{1}\right]}_{W_{A_{1}}\left(p_{n}+q_{2}\right)} \cdot \ldots \cdot \underbrace{\operatorname{det}\left[A_{1}-\left(p_{n}+q_{p}\right) I_{1}\right]}_{W_{A_{1}}\left(p_{n}+q_{p}\right)}
\end{aligned}
$$

so

$$
\operatorname{det} A_{3}=\left(\lambda_{1} \cdot \lambda_{2} \cdot \ldots \cdot \lambda_{m}\right)^{m n p} \cdot W
$$

where

$$
\begin{aligned}
W= & \left(1-\frac{p_{1}+q_{1}}{\lambda_{1}}\right) \cdot \ldots \cdot\left(1-\frac{p_{1}+q_{1}}{\lambda_{m}}\right) \cdot \ldots \cdot\left(1-\frac{p_{n}+q_{1}}{\lambda_{1}}\right) \cdot \ldots \cdot\left(1-\frac{p_{n}+q_{1}}{\lambda_{m}}\right) \cdot \\
& \cdot\left(1-\frac{p_{1}+q_{2}}{\lambda_{1}}\right) \cdot \ldots \cdot\left(1-\frac{p_{1}+q_{2}}{\lambda_{m}}\right) \cdot \ldots \cdot\left(1-\frac{p_{n}+q_{2}}{\lambda_{1}}\right) \cdot \ldots \cdot\left(1-\frac{p_{n}+q_{2}}{\lambda_{m}}\right) \cdot \\
& \cdot\left(1-\frac{p_{1}+q_{p}}{\lambda_{1}}\right) \cdot \ldots \cdot\left(1-\frac{p_{1}+q_{p}}{\lambda_{m}}\right) \cdot \ldots \cdot\left(1-\frac{p_{n}+q_{p}}{\lambda_{1}}\right) \cdot \ldots \cdot\left(1-\frac{p_{n}+q_{p}}{\lambda_{m}}\right) . \\
= & 1-S_{1}+S_{2}-\ldots+(-1)^{m n p} S_{m n p}
\end{aligned}
$$


where $S_{1}$ (the first symmetric polynomial of the indicated above variables) is equal to

$$
\begin{aligned}
& S_{1}=\sum_{\substack{1 \leq \alpha \leq m \\
1 \leq \beta \leq n \\
1 \leq>\leq p}} \frac{p_{\beta}+q_{\gamma}}{\lambda_{\alpha}}= \\
& =n p\left(\frac{1}{\lambda_{1}}+\frac{1}{\lambda_{2}}+\ldots+\frac{1}{\lambda_{m}}\right)(\underbrace{p_{1}+p_{2}+\ldots+p_{n}}_{\omega_{1}})(\underbrace{q_{1}+q_{2}+\ldots+q_{p}}_{\eta_{1}})= \\
& =n p \frac{\tau_{m-1}}{\tau_{m}} \cdot \omega_{1} \cdot \eta_{1}
\end{aligned}
$$

$\left(\tau_{j}=\tau_{j}\left(\lambda_{1}, \lambda_{2}, \ldots, \lambda_{m}\right)\right.$ - fundamental symmetric polynomials $\left.1 \leq j \leq m\right)$.

The other symmetric polynomials $S_{2}, \ldots, S_{m n-1}$ we calculate from the Newton formulas [3].

Next we calculate $S_{2}$

$$
S_{2}=\frac{1}{2}\left|\begin{array}{ll}
\sigma_{1} & 1 \\
\sigma_{2} & \sigma_{1}
\end{array}\right|=\frac{1}{2}\left(\sigma_{1}^{2}-\sigma_{2}\right)=\frac{1}{2}\left(S_{1}^{2}-\sigma_{2}\right)
$$

where $\sigma_{1}=S_{1}$ and $\sigma_{2}$ is the power polynomials of degree 2 .

We have

$$
\begin{aligned}
& \sigma_{2}=n p\left(\frac{1}{\lambda_{1}^{2}}+\frac{1}{\lambda_{2}^{2}}+\ldots+\frac{1}{\lambda_{m}^{2}}\right) \\
& {\left[\left(p_{1}+q_{1}\right)^{2}+\ldots+\left(p_{n}+q_{1}\right)^{2}+\left(p_{1}+q_{2}\right)^{2}+\ldots+\left(p_{n}+q_{2}\right)^{2}+\ldots\right.} \\
& \left.+\left(p_{1}+q_{p}\right)^{2}+\ldots+\left(p_{n}+q_{p}\right)^{2}\right]=n p\left(\frac{1}{\lambda_{1}^{2}}+\frac{1}{\lambda_{2}^{2}}+\ldots+\frac{1}{\lambda_{m}^{2}}\right) \cdot \tilde{\sigma}_{2}
\end{aligned}
$$

where

$$
\begin{aligned}
& \tilde{\sigma}_{2}=p\left(p_{1}^{2}+\ldots+p_{n}^{2}\right)+2\left(p_{1}+\ldots+p_{n}\right)\left(q_{1}+\ldots+q_{p}\right)+n\left(q_{1}^{2}+\ldots+q_{p}^{2}\right) \\
& =p\left(\omega_{1}^{2}-2 \omega_{2}\right)+2 \omega_{1} \eta_{1}+n\left(\eta_{1}^{2}-2 \eta_{2}\right)
\end{aligned}
$$

and finally

$$
\begin{aligned}
& S_{2}=\frac{1}{2}\left(S_{1}^{2}-\sigma_{2}\right)= \\
& =\frac{1}{2}\left[S_{1}^{2}-n p\left(\frac{1}{\lambda_{1}^{2}}+\frac{1}{\lambda_{2}^{2}}+\ldots+\frac{1}{\lambda_{m}^{2}}\right) \cdot \tilde{\sigma}_{2}\right]=\frac{1}{2}\left[S_{1}^{2}-n p \frac{\tau_{m-1}^{2}}{\tau_{m-1}^{2}}-\frac{2 \tau_{m-2}}{\tau_{m}} \cdot \tilde{\sigma}_{2}\right]
\end{aligned}
$$


The last component of expression $W$ is equal to

$$
\begin{aligned}
& S_{m n p}=\left(\frac{1}{\lambda_{1} \lambda_{2} \cdot \ldots \cdot \lambda_{m}}\right)^{m n p}\left[\left(p_{1}+q_{1}\right)^{m} \cdot \ldots \cdot\left(p_{n}+q_{1}\right)^{m} \cdot\right. \\
& \left.\cdot\left(p_{1}+q_{2}\right)^{m} \cdot \ldots \cdot\left(p_{n}+q_{2}\right)^{m} \cdot . .\left(p_{1}+q_{p}\right)^{m} \cdot \ldots \cdot\left(p_{n}+q_{p}\right)^{m}\right]= \\
& =\left(\frac{1}{\lambda_{1} \lambda_{2} \cdot \ldots \cdot \lambda_{m}}\right)^{m n p}\left[\left(p_{1}+q_{1}\right) \cdot \ldots \cdot\left(p_{n}+q_{1}\right) \cdot\left(p_{1}+q_{2}\right) \cdot \ldots \cdot\left(p_{n}+q_{2}\right) \cdot \ldots\right. \\
& \left.\cdot\left(p_{1}+q_{p}\right) \cdot \ldots \cdot\left(p_{n}+q_{p}\right)\right]^{m}=\left(\frac{1}{\lambda_{1} \lambda_{2} \cdot \ldots \cdot \lambda_{m}}\right)^{m n p} \cdot\left(\tilde{S}_{m n p}\right)^{m}
\end{aligned}
$$

where

$$
\begin{aligned}
& \tilde{S}_{m n p}= \\
& =\left(p_{1}+q_{1}\right) \cdot \ldots \cdot\left(p_{n}+q_{1}\right) \cdot\left(p_{1}+q_{2}\right) \cdot \ldots \cdot\left(p_{n}+q_{2}\right) \cdot\left(p_{1}+q_{p}\right) \cdot \ldots \cdot\left(p_{n}+q_{p}\right)= \\
& =\left(p_{1} \cdot \ldots \cdot p_{n}\right)^{n} \cdot\left(1+\frac{q_{1}}{p_{1}}\right) \cdot \ldots \cdot\left(1+\frac{q_{1}}{p_{n}}\right) \cdots \ldots \cdot\left(1+\frac{q_{2}}{p_{1}}\right) \cdot \ldots \cdot\left(1+\frac{q_{2}}{p_{n}}\right) . \\
& \cdot \ldots \cdot\left(1+\frac{q_{p}}{p_{1}}\right) \cdot \ldots \cdot\left(1+\frac{q_{p}}{p_{n}}\right)= \\
& =\left(p_{1} \cdot \ldots \cdot p_{n}\right)^{n} \cdot\left(1+\hat{S}_{1}+\hat{S}_{2}+\ldots+\hat{S}_{m p}\right)
\end{aligned}
$$

and so

$$
\begin{aligned}
& \hat{S}_{1}=\left(\frac{1}{p_{1}}+\ldots+\frac{1}{p_{n}}\right)\left(q_{1}+\ldots+q_{p}\right)=\frac{\omega_{n-1}}{\omega_{n}} \eta_{1} \\
& \hat{S}_{2}=\left(\frac{\omega_{n-1}^{2}}{\omega_{n}^{2}}-2 \frac{\omega_{n-2}}{\omega_{n}}\right)\left(\eta_{1}^{2}-2 \eta_{2}\right) \\
& \hat{S}_{m p}=\frac{1}{\left(q_{1} \cdot \ldots \cdot q_{p}\right)^{n}}\left(p_{1} \cdot \ldots \cdot p_{n}\right)^{p}=\frac{\eta_{1}^{n}}{\omega_{1}^{k}}
\end{aligned}
$$

Moreover, in equation (24) we assume $j \leq E\left(\frac{m p-1}{2}\right)$, because the following property takes place [4]: 


$$
\begin{aligned}
& \hat{S}_{m p-j}\left(\frac{q_{1}}{p_{1}}, \ldots, \frac{q_{1}}{p_{n}} ; \frac{q_{2}}{p_{1}}, \ldots, \frac{q_{2}}{p_{n}} ; \ldots ; \frac{q_{p}}{p_{1}}, \ldots, \frac{q_{p}}{p_{n}}\right)= \\
& =\hat{S}_{j}\left(\frac{p_{1}}{q_{1}}, \ldots, \frac{p_{1}}{q_{p}} ; \frac{p_{2}}{q_{1}}, \ldots, \frac{p_{2}}{q_{p}} ; \ldots ; \frac{p_{n}}{q_{1}}, \ldots, \frac{p_{n}}{q_{p}}\right) \cdot \hat{S}_{m p}
\end{aligned}
$$

For example

$$
\begin{aligned}
& \hat{S}_{m p-1}\left(\frac{q_{1}}{p_{1}}, \ldots, \frac{q_{1}}{p_{n}} ; \frac{q_{2}}{p_{1}}, \ldots, \frac{q_{2}}{p_{n}} ; \ldots ; \frac{q_{p}}{p_{1}}, \ldots, \frac{q_{p}}{p_{n}}\right)= \\
& =\hat{S}_{1}\left(\frac{q_{1}}{p_{1}}, \ldots, \frac{q_{1}}{p_{n}} ; \frac{q_{2}}{p_{1}}, \ldots, \frac{q_{2}}{p_{n}} ; \ldots ; \frac{q_{p}}{p_{1}}, \ldots, \frac{q_{p}}{p_{n}}\right) \cdot \hat{S}_{m p}= \\
& =\frac{\eta_{p-1}}{\eta_{p}} \omega_{1} \frac{\eta_{1}^{n}}{\omega_{1}^{k}}=\frac{\eta_{p-1} \eta_{1}^{n}}{\eta_{p} \omega_{1}^{k-1}}
\end{aligned}
$$

Let us note also, that in calculating $\hat{S}_{j}$ the following sum appears

$$
\begin{aligned}
& \left(p_{1}+q_{1}\right)^{j}+\ldots+\left(p_{n}+q_{1}\right)^{j}+\left(p_{1}+q_{2}\right)^{j}+\ldots+\left(p_{n}+q_{2}\right)^{j}+\ldots \\
& +\left(p_{1}+q_{p}\right)^{j}+\ldots+\left(p_{n}+q_{p}\right)^{j}= \\
& =\left[p_{1}^{j}+\left(\begin{array}{c}
j \\
1
\end{array}\right) p_{1}^{j-1} q_{1}+\ldots+q_{1}^{j}\right]+\ldots+\left[p_{n}^{j}+\left(\begin{array}{c}
j \\
1
\end{array}\right) p_{n}^{j-1} q_{1}+\ldots+q_{1}^{j}\right]+ \\
& +\left[p_{1}^{j}+\left(\begin{array}{c}
j \\
1
\end{array}\right) p_{1}^{j-1} q_{2}+\ldots+q_{2}^{j}\right]+\ldots+\left[p_{n}^{j}+\left(\begin{array}{l}
j \\
1
\end{array}\right) p_{n}^{j-1} q_{2}+\ldots+q_{2}^{j}\right]+ \\
& +\left[p_{1}^{j}+\left(\begin{array}{c}
j \\
1
\end{array}\right) p_{1}^{j-1} q_{p}+\ldots+q_{p}^{j}\right]+\ldots+\left[p_{n}^{j}+\left(\begin{array}{c}
j \\
1
\end{array}\right) p_{n}^{j-1} q_{p}+\ldots+q_{p}^{j}\right]= \\
& =p \underbrace{\left(p_{1}^{j}+\ldots+p_{n}^{j}\right)}_{\sigma_{j}^{o}}+\left(\begin{array}{l}
j \\
1
\end{array}\right) \underbrace{\left(p_{1}^{j-1}+\ldots .+p_{n}^{j-1}\right)}_{\sigma_{j-1}^{\infty}} \underbrace{\left(q_{1}+\ldots+q_{p}\right)}_{\sigma_{1}^{\eta}}+\ldots+ \\
& +n \underbrace{\left(q_{1}^{j}+\ldots+q_{p}^{j}\right)}_{\sigma_{j}^{n}}
\end{aligned}
$$

The power series polynomials $\sigma_{k}^{\omega}$ and $\sigma_{l}^{\eta}, 1 \leq k, l \leq j$, appearing in the above expression are calculated in a known way with Newton's formulas [4]. For the calculation of $\omega, \eta$ and $\tau$ we use the formulas from [4]. 
According to the known method described in [4], we obtain the values

$$
\begin{aligned}
\tau_{j} & =\left(\begin{array}{c}
m \\
j
\end{array}\right) a^{j}-\left(\begin{array}{c}
m-1 \\
1
\end{array}\right)\left(\begin{array}{c}
m-2 \\
j-2
\end{array}\right) a^{j-2}+ \\
& +\left(\begin{array}{c}
m-2 \\
2
\end{array}\right)\left(\begin{array}{c}
m-4 \\
j-4
\end{array}\right) a^{j-4}-\left(\begin{array}{c}
m-3 \\
3
\end{array}\right)\left(\begin{array}{c}
m-6 \\
j-6
\end{array}\right) a^{j-6}+\ldots
\end{aligned}
$$

and

$$
\omega_{k}= \begin{cases}0 & \text { if } k \text { is even } \\
(-1)^{k / 2}\left(\begin{array}{c}
n-(k / 2) \\
k / 2
\end{array}\right) & \text { if } k \text { is odd }\end{cases}
$$

(from the formula (12)) and

$$
\eta_{l}= \begin{cases}0 & \text { if } l \text { is even } \\
(-1)^{l / 2}\left(\begin{array}{c}
p-(l / 2) \\
l / 2
\end{array}\right) & \text { if } l \text { is odd }\end{cases}
$$

(from the formula (13)).

\section{Remark}

The procedure given above constitutes a introduction to the general procedure for calculating the determinants of the matrix block in the $n$-dimensional case. The $n$-dimensional case will be considered in the next article.

\section{References}

[1] Biernat G., Lara-Dziembek S., Pawlak E., The determinants of the three-band block matrices, Scientific Research of the Institute of Mathematics and Computer Science 2012, 3(11), 5-8.

[2] Biernat G., Lara-Dziembek S., Pawlak E., The determinants of the block band matrices based on the $n$-dimensional Fourier equation. Part 1, Scientific Research of the Institute of Mathematics and Computer Science 2013, 3(12), 5-13.

[3] Mostowski A., Stark M., Elementy algebry wyższej, PWN, Warszawa 1975.

[4] Biernat G., Lara-Dziembek S., Pawlak E., Symmetric polynomials in the 2D Fourier equation, Journal of Applied Mathematics and Computational Mechanics 2014, 4(13), 5-12. 